\title{
ADDENDUM \\ Using satellites to probe extrasolar planet formation
}

\author{
Paul Withers ${ }^{1}$ and Jason W. Barnes ${ }^{2}$ \\ ${ }^{1}$ Department of Astronomy, Boston University, \\ 725 Commonwealth Avenue, Boston, MA 02215, USA \\ email: withers@bu.edu \\ ${ }^{2}$ Department of Physics, University of Idaho, \\ 875 Perimeter Drive, Moscow, ID 83844-0903, USA \\ email: jwbarnes@uidaho.edu
}

\begin{abstract}
Planetary satellites are an integral part of the hierarchy of planetary systems. Here we make two predictions concerning their formation. First, primordial satellites, which have an array of distinguishing characteristics, form only around giant planets. If true, the size and duration of a planetary system's protostellar nebula, as well as the location of its snow line, can be constrained by knowing which of its planets possess primordial satellites and which do not. Second, all satellites around terrestrial planets form by impacts. If true, this greatly enhances the constraints that can be placed on the history of terrestrial planets by their satellites' compositions, sizes, and dynamics.
\end{abstract}

Keywords. planets and satellites: formation, stars: formation, planetary systems: formation

Here we explore how the existence and nature of a planet's satellite system can be used to constrain how the planet formed. We make two predictions concerning the formation of planetary satellites and investigate their consequences. They outline the potential significance of future discoveries of satellites for extrasolar planets for the histories and current states of these satellites, planets, and planetary systems. Although recent observations of extrasolar planetary systems have drastically reshaped ideas of planet formation, models still predict a bimodal distribution of volatile-rich planets analogous to giant planets and refractory-rich planets analogous to terrestrial planets (Reipurth et al. 2007). Since satellites are abundant in the solar system, we expect that satellites are likely to be present around many extrasolar planets - as long as they have not been lost due to tidal evolution (Barnes \& O'Brien 2002). Several theories of satellite formation have been proposed to explain the diverse satellites within the solar system, including in situ formation in a protoplanetary disk, gravitational capture, atmospheric capture, fission, and the impact between a planet and another body (Stevenson et al. 1986). These mechanisms are also candidates for how extrasolar satellites form.

Prediction 1: Satellites that formed in the same place and at the same time as their parent planet exist only around planets analogous to giant planets. The distinguishing characteristics of such satellites are: low eccentricity, prograde orbits near the planet's equatorial plane and well within the planet's Hill sphere; same age of formation as the parent planet; and elemental and isotopic compositions that, although potentially modified by accretion and subsequent processes, are related to the environmental conditions at the location and time of the formation of the parent planet. Extrasolar planets with satellites that possess these characteristics are predicted to be volatile-rich. That provides a constraint on relationships between the planet's mass, density, size, temperature 
and spectrum. They are also predicted to have formed rapidly within a protoplanetary disk. Since their elemental and isotopic composition reflects conditions where they formed, trends in these compositions can be used to determine spatial variations in the protostellar nebula and to constrain planetary migration post-formation. Mapping the distribution of such planets within a planetary system places constraints on the size and duration of the protostellar nebula from which the star, planets and satellites formed. If this prediction is disproven, then the presence of a protoplanetary disk during planet formation is not a discriminant between volatile-rich giant planets and refractory-rich terrestrial planets, which has substantial implications for planet formation.

Prediction 2: All satellites above a threshold mass around planets analogous to terrestrial planets formed by the accretion of ejecta from an impact. The distinguishing characteristics of satellites formed from the accretion of impact ejecta are (Hartmann et al. 1986, Stern et al. 1997, Canup \& Righter 2000): low abundance of siderophile constituents relative to the parent planet; low relative abundance of volatiles; large satellite to planet mass ratio; large ratio of the orbital angular momentum of the satellite to the total angular momentum of the system; and isotopic composition that, although potentially modified by subsequent processes, suggests a common origin for the material in the planet and satellite. Extrasolar planets with satellites that possess these characteristics are predicted to be refractory-rich. This provides a constraint on relationships between the planet's mass, density, size, temperature and spectrum. They are also predicted to have formed slowly from the accretion of refractory planetesimals. Due to mixing of planetesimals, the elemental and isotopic composition of such planets reflects conditions across a broad region of the protostellar nebula. Mapping the distribution of such planets within a planetary system places constraints on the size and duration of the protostellar nebula from which the star, planets and satellites formed. If this prediction is disproven, then either at least one seemingly impractical mechanism for satellite formation must occur more easily than is currently thought or an unsuspected mechanism for satellite formation exists. Either case has substantial implications for satellite formation.

A meaningful planetary classification scheme should be based on currently observable characteristics, yet also be related to planetary formation and history. Planets in the mass range $1 \mathrm{M}_{\oplus} \leqslant M \leqslant 10 \mathrm{M}_{\oplus}$ could either be "mini-Neptunes", like GJ1214b (Charbonneau et al. 2009) or "super-Earths" like CoRoT-7b (Léger et al. 2009). Future discoveries of planets in this mass range are likely to blur the currently clear boundary between terrestrial planets and giant planets. Consideration of a planet's retinue of satellites, particularly primordial satellites, may help fix the boundaries of planetary classification schemes.

\section{References}

Barnes, J. W. \& O'Brien, D. P. 2002, ApJ, 575, 1087

Canup, R. M. \& Righter, K. 2000, Origin of the Earth and Moon (Tucson, Arizona: Univ. Arizona Press)

Charbonneau, D., et al. 2009, Nature, 462, 891

Hartmann, W. K., Phillips, R. J., \& Taylor, G. J. 1986, Origin of the Moon (Houston, Texas: Lunar and Planetary Institute)

Léger, R., et al. 2009, A\&A, 506, 287

Reipurth, B., Jewitt, D., \& Keil, K. 2007, Protostars and Planets V (Tucson, Arizona: Univ. Arizona Press)

Stern, A. S., McKinnon, W. B., \& Lunine, J. I. 1997, in A.S. Stern \& D.J. Tholen (eds.), Pluto and Charon (Tucson, Arizona: Univ. Arizona Press), 605

Stevenson, D. A., Harris, A. W., \& Lunine, J. I. 1986, in: J.A. Burns \& M.S. Matthews (eds.), Satellites (Tucson, Arizona: Univ. Arizona Press), 39 